\title{
Stretchable bioelectronics for medical devices and systems
}

Ghaffari, Roozbeh, rghaffari@mc10inc.com, MC1 Inc.

\begin{abstract}
Advances in the microelectronics and telecommunications industries have driven important breakthroughs in medical technologies and health diagnostics over the past decade. However, there are fundamental gaps in size, sensing modalities and mechanical properties between the standard rigid electronics, employed in medical devices today, and the signals emitted by soft biological structures. Here, the author describes novel materials, mechanics, and designs for emerging classes of health monitoring systems and invasive medical devices, including soft wearable patches and flexible catheter-based systems. These emerging devices incorporate microfabricated arrays of sensors (e.g., dry electrodes, temperature sensors, and accelerometers), actuators (e.g., micro-LEDs, piezo-electric ribbons, pacing electrodes), and silicon nanomembrane semiconductors, configured in ultrathin, flexible formats for continuous monitoring, therapy delivery, and energy harvesting. Quantitative analyses of strain distributions and circuit performances under stress illustrate the ability of these systems to mechanically couple with moist soft biological tissues, in a way that is mechanically invisible to the target biological substrate and comfortable for the patient. As demonstrations of this technology, the author presents representative examples of flexible and stretchable systems for use in both noninvasive and minimally invasive applications, which leverage the same class of microfabricated circuits and flexible sensor arrays. The fabrication strategies and design concepts described in this discussion can be tailored to various biological substrates and geometries of interest, and thus have the potential to broadly bridge the gap that exists between rigid electronics and biology.
\end{abstract}

